\title{
Discussion - Populations of OB stars in galaxies
}

\author{
Gregg A. Wade ${ }^{1}$ and Hideyuki Saio ${ }^{2}$ \\ ${ }^{1}$ RMC, Kingston, Canada \\ ${ }^{2}$ Astronomical Institute, Graduate School of Science, Tohoku University, Sendai, \\ Miyagi 980-8578, Japan
}

\begin{abstract}
This article summarises the discussion following Session 3 (Populations of OB stars in galaxies) of IAUS 272 .
\end{abstract}

This discussion followed talks by N. Langer (The elusive massive main sequence stars), T. Decressin (Massive stars in globular clusters: drivers of chemical and dynamical evolution), C. Evans (Populations of OB stars in galaxies), C. Martayan (Populations of Be stars: stellar evolution of extreme stars), A. Bonanos (Infrared properties of Active OB stars in the Magellanic Clouds from the Spitzer SAGE Survey), A. Mironichenko (The $\mathrm{B}[\mathrm{e}]$ phenomenon in the Milky Way and Magellanic Clouds) and D. Bomans (Massive variable stars at very low metallicity?).

J. Zorec: Comment related to presentations by Martayan / Maeder / Miroschnichenko. The problems of abundance determination in fast rotators (Be stars) is challenging. First, massive Be stars are required to get lines of $\mathrm{CNO}$ in the optical. In addition, these stars should be viewed pole-on to reduce $v \sin i$ and produce sharp lines. One must use models including rotational effects, otherwise one will tend to overestimate the $\mathrm{N}$ abundance. Veiling effects are potentially a problem, and need to be corrected, in particular for poleon stars because it is for these objects that we have the largest contribution from the (face-on) disc. The calibration presented by Martayan is only one of several possible. Uncertainties result from lack of knowledge of intrinsic magnitude of "naked" star (i.e. there is a contribution form the disc). Also, it is not clear what the structure of envelope does to the scatter. So positions of stars on that diagram are uncertain.

G. Peters: Comments concerning abundances/bipolar structures of Be stars. UV avoids veiling problems, and various $\mathrm{C}$ and $\mathrm{O}$ lines available. But emission lines are present as well, usually in doubly-ionised lines (e.g. Si II and IV can be fit well, but not Si III). This emission consitutes evidence for external plasma at temperatures higher than the disc $(20 \mathrm{kK})$, probably from a bipolar flow. Concerning $v \sin i$ : has personally found vsini's from UV features to be slightly smaller than in the optical, possibly due to temperature variation of photosphere from pole to equator. Discussion of binary and binary fraction for Be stars. One issue not discussed in the talks is that very close binaries have not been examined. With Gies, has found subdwarf companions around 2 objects. Also Be counterparts of Algol systems, etc.

J. Puls: A completely different topic: what is the present state of understanding $\mathrm{B}[\mathrm{e}]$ supergiants? Question directed to the evolutionary people, there are always two scenarios discussed. Do these result from binary merger or post-supergiant evolution?

A. Maeder: if such a star rotates quite fast, then it should have a fast low density polar wind, and a slow, high density equatorial wind where dust can form, so this would be consistent with the "peanut" shape. The presence of companion may also be possible, but 
why would a companion make the polar ejection? Is the frequency of mergers compatible with the observed number of such stars? Perhaps this is not sufficiently frequent?

Inaudible question.

A. Mironichenko: From the observational point of view, about $10 \mathrm{~B}[\mathrm{e}]$ supergiants confirmed in the galaxy. Most are binaries not yet merged. For example, in my talk I showed B1 supergiant $+\mathrm{F}$ supergiant, with a period of about $1 / 2$ year. So most are actually in binary systems.

N. Langer: A few comments in this respect. One B[e] supergiant for which strong evidence exists that merger was the source, R4 in SMC. Still is a binary, has an main sequence A star companion $\left(5 M_{\odot}\right)$, so B star should have long been gone, so must be from merger. Evidence for a merger in this case is quite evident. From binary population synthesis statistics, $10 \%$ of all MS stars undergo mergers during their MS evolution - a rather high fraction of stars can be expected to merge. Concerning Be stars: the binary incidence is very important. In Be X-ray binaries, all the B-type main sequence stars are Be stars, so binary is related to Be phenomenon. If a neutron star is formed in a supernova there will be a very strong kick given to the neutron star and ejection from the binary system, so most Be X-ray binaries should have been disrupted. It is expected that $95 \%$ of such systems get disrupted. Therefore for each Be X-ray binary that we see with a neutron star companion, there should be many single Be stars (that were originally in binary systems, but from which the neutron star has been ejected).

O. Chesneau: Regarding B[e] stars: From an observer's point of view, the formation of disk is a consequence of binarity, perhaps without a merger. In the case of a merger, perhaps the disc was formed before merger, and is not a consequence of merger. It is difficult to imagine disc as a consequence of merger - how to form long-lived Keplerian dense, dusty disc? No explanation has been offered except binary. This conclusion is unavoidable in the planetary nebula community - you are obliged to invoke angular momentum input from a companion, even if it is just a 10 Jupiter-mass planet.

M. Smith: Topic that could not be fit into one of the talks, but appropriate in this context: environment of central black hole of Milky Way. IR studies show that there are several B stars orbiting within a few AU of Milky Way's central black hole. Where do these stars come from? How did they form?

D. Baade: Recent workshop in Garching about nuclear star clusters. The clusters form in gas flow around black hole (stars are so young they must have formed in situ). A relationship exists between the mass of the cluster and velocity dispersion of parent galaxy (and hence the mass of the black hole). Related to feedback in the formation of galaxies on various scales.

(Inaudible discussion)

G. Meynet: A point that deserves further explanation and needs to be explained by models, is non-continuous formation of Be star discs - produced in discrete mass loss events. To know the timescales is important to understand physics behind the phenomenon. If it is due to critical velocity, we would expect this kind of back-and-forth evolution as it reaches $v_{\text {crit }}$, loses mass, slows down, etc. Would a binary be able to produce this kind of episodic phenomenon in the injecton of matter into the disc?

C. Martayan: Two comments: First about Langer's remark. In the case of binary disruption, shouldn't we find Be stars as runaways, or associated with supernova remnants, if this were true? But most are found in open clusters. Consider $\delta$ Sco which undergoes mass ejection when companion passes periastron. So this shows at least one connection to binarity, but may not be the case for all Be stars.

N. Langer: In the binary scenario, you spin up a B star by accretion (from the companion?) and left with a rapidly-rotating star. How is this different from a single star that 
is born rapidly rotating? Regarding Be stars / Be X-ray binaries: supernova remnants might still be around, but lifetime is just $10^{4}-10^{5}$ years, so only small fraction of stars would be expected to show that. Need statistics to test this. What about them being runaway stars from the supernova explosion? In principle right, but many systems are wide binaries. Perhaps through conservative mass transfer they may evolve to very wide orbits which are relatively immune to generating runaways.

D. Baade: You are right, there is no a priori reason to believe that single star and member of binary system would evolve differently after spin-up has occurred. However, this does not necessarily make them both Be stars: what is the 2nd (or 3rd or 4th) ingredient required? e.g. if pulsation is important, then internal structure must also be the same.

A. Maeder: It is sure that a supernova remnant has limited lifetime. A critical parameter for merging is for how long do we see polar ejection and disc? Also question of lifetime of the merging phenomenon. Not sure it is much longer than remnant lifetime. N. Langer, do you have an idea of the lifetime of the events associated with a merging event, e.g. the peculiarities associated with that?

N. Langer: Well, once the stars merge, they live on as a rapidly rotating single star for a long time. Whether or not it is a Be star or not depends on D. Baade's remark, that there may be factors involved that we do not know.

W. Huang: Recent result of B star rotation survey. Low vs. high mass B stars behave differently.

G. Peters: A phenomenon that is known more in the binary community is a class of Algol binaries that show long-term cycles of activity. Orbits are of order 10 days, but cycles are 6 months- 2 years in the apparent mass transfer activity. Mass transfer in these systems seems to vary cyclically on long timescales. Perhaps relevant to discussion of binarity?

S. Owocki: To follow up on George's and Dietrich's comments: we should distinguish between $\omega / \omega_{\text {crit }}$ and $v / v_{\text {crit }}$. To launch material with just thermal energy from stellar surface you need $v / v_{\text {crit }} 95 \%$, but this is $\omega / \omega_{\text {crit }}$ of around $98-99 \%$. So must be very close to critical to use just thermal energy. Pulsation could allow ejection at lower values of $v / v_{\text {crit }}$. If there is no process that forces the star to continually spin up to approach critical (e.g. internal evolution) the ejection process would only occur once. So such internal evolution much occur to continually push star toward critical, as we see episodic ejections. 